\title{
Initial Conditions, Generalized Functions, and the Laplace Transform
}

\author{
Troubles at the origin \\ Kent H. Lundberg, Haynes R. Miller, and David L. Trumper \\ Massachusetts Institute of Technology \\ Version 5.5
}

The unilateral Laplace transform is widely used to analyze signals, linear models, and control systems, and is consequently taught to most engineering undergraduates. In our courses at MIT in the departments of electrical engineering and computer science, mathematics, and mechanical engineering, we have found some significant pitfalls associated with teaching our students to understand and apply the Laplace transform. We have independently concluded that one reason students find the Laplace transform difficult is that there are significant confusions present in many of the standard textbook presentations of this subject, in all three of our disciplines.

A key issue is the treatment of the origin. Many texts [1]-[5] define the Laplace transform of a time function $f(t)$ as

$$
\mathcal{L}\{f(t)\}=\int_{0}^{\infty} f(t) e^{-s t} d t
$$

without specifying the meaning of the lower limit of integration. Said informally, does the integral include the origin fully, partially, or not at all? This question becomes critical when singularities - the delta function and its derivatives-occur at $t=0$.

In the context of differential equations, singularities arise from discontinuites in signals that are differentiated, or from considering systems driven with singularities in the input. Since the analysis of transient response is one of the key topics in dynamic-systems courses, we need to teach techniques that handle discontinuities and singularities, including those at the origin. In particular, any presentation based on the unilateral Laplace transform should unambiguously specify how the origin is treated.

To understand and apply the unilateral Laplace transform, students need to be taught an approach that addresses arbitrary inputs and initial conditions. Some mathematically oriented treatments of the unilateral Laplace transform, such as [6] and [7], use the $\mathcal{L}_{+}$form

$$
\mathcal{L}_{+}\{f(t)\}=\int_{0^{+}}^{\infty} f(t) e^{-s t} d t
$$

with the associated differentiation identity

$$
\mathcal{L}_{+}\left\{f^{\prime}(t)\right\}=s F(s)-f\left(0^{+}\right) .
$$

However, with this $\mathcal{L}_{+}$form, the transform of the unit impulse is zero! This result is often puzzling to students, who rightly expect the impulse response of a linear system to be nonzero.
Alternatively, the $\mathcal{L}_{-}$Laplace transform is given by

$$
\mathcal{L}_{-}\{f(t)\}=F(s)=\int_{0^{-}}^{\infty} f(t) e^{-s t} d t,
$$

where the domain of integration fully includes the origin and any singularities occuring at that time. This form is adopted in many texts, such as [8]-[12]. The definition (3) of the Laplace transform implies the time-derivative rule

$$
\mathcal{L}_{-}\left\{f^{\prime}(t)\right\}=s F(s)-f\left(0^{-}\right),
$$

whereby initial conditions existing before $t=0$ are brought into the analysis. We refer to $f\left(0^{-}\right)$as the pre-initial value of $f$. In the context of differential equations this term is the pre-initial condition.

With either (1) or (3) as the definition of the Laplace transform, the initial-value theorem is

$$
\lim _{s \rightarrow \infty \cdot 1} s F(s)=f\left(0^{+}\right),
$$

involving the post-initial value at $t=0^{+}$, where the notation $s \rightarrow \infty \cdot 1$ indicates that the limit is taken along the positive real axis.

Many books [13]-[17] implicitly adopt the $\mathcal{L}_{+}$transform by using the derivative property (2), but are not explicit as to the lower limit on the Laplace integral. However, these texts still find that the transform of the unit impulse is $\mathcal{L}\{\delta(t)\}=1$. In the engineering context, this inconsistency can likely be traced back to the classic text [18], which is cited by the landmark control texts [19]-[23]. The resulting conflict is frequently circumvented by defining the delta function as occurring completely to the right of $t=0$ [15], [24]-[26]. If the delta function occurs to the right of the origin, what then does $0^{+}$ mean, and what time value is associated with the initialvalue theorem (5)? With such questions left unaddressed, it is difficult to teach engineering students to reliably analyze transients at the origin in dynamic systems with the $\mathcal{L}_{+}$ transform. Additionally, with the $\mathcal{L}_{+}$approach the post-initial value $f\left(0^{+}\right)$needs to be determined by independent means. We certainly owe it to students not to leave this issue fuzzy by simply avoiding problems that require a discussion of $0^{-}$ and $0^{+}$.

These troubles arise from a reluctance to use the generalized derivative. One of the goals of this article is to show that a formal but easily understood treatment can be based on generalized functions. In brief, the fact that the derivative at a 
discontinuity produces a delta function must be embraced from the outset. The subtitle of this article, "Troubles at the origin," was inspired by Section 3-6 of [27], which identifies the necessity of distinguishing between $0^{-}$and $0^{+}$. However, the subsequent reasoning in [27], which relies on the definition of an alternative impulse function $\delta_{+}(t)$, reaches the conclusion that the $\mathcal{L}_{+}$transform is preferable and that $\delta(t)$ at the origin should be ignored. The $\delta_{+}(t)$ approach is also used by [28]. By disguising the fact that there is a discontinuity at the origin, such treatments give special treatment to to $t=0$ and complicate the task of distinguishing between functions such as $f(t)=1$ and the unit-step function, which have the same Laplace transform and the same value $f\left(0^{+}\right)$but which have different derivatives. In addition, this $\delta_{+}(t)$ function lacks any physical interpretation as an impulse, and we know of no theory of distributions that includes one-sided singularity functions; introducing them represents a significant departure from standard mathematical theory.

While it is not possible to devote full attention to generalized functions in a typical undergraduate course, this confusion surrounding $0^{-}$and $0^{+}$- this "skeleton in the closet" as [10] calls it - needs to be brought out fully into the light. The goal of this article is to highlight Laplace transform definitions and properties that allow a readily taught and correct analysis of dynamic systems incorporating nonzero initial conditions and where the system is driven by signals that include singularity functions at the origin. To this end, we advocate the use of the $\mathcal{L}_{-}$Laplace transform (3), along with its derivative rule (4). We present reasonable mathematical support for this approach, along with an accessible theory of generalized functions, including how singularity functions are defined, how these functions are combined with regular functions to form generalized functions, and how mathematical operations on these generalized functions can be consistently defined. This treatment is suitable for most engineering uses.

The occurence of $0^{-}$in (4) commits us to specifying the value $f\left(0^{-}\right)$and, if we wish to differentiate further, the values $f^{(n)}\left(0^{-}\right)$as well, where $f^{(n)}$ indicates the $n^{\text {th }}$ derivative. With this viewpoint, initial conditions are more clearly defined as pre-initial conditions. This extension of the data inherent in a function provides a natural representation of pre-initial conditions, describing the state "just before $t=0$." In many applications the signal $f(t)$ is assumed to be zero for $t<0$; this choice represents the rest pre-initial conditions $f^{(n)}\left(0^{-}\right)=0$ for all $n$. In this case, the derivative rule takes on the simpler form $\mathcal{L}_{-}\left\{f^{\prime}(t)\right\}=s F(s)$. However, nonzero preinitial conditions are frequently encountered, and thus need to be addressed systematically in any transform approach.

With this overview in place, the remainder of this article is organized as follows: We first motivate the discussion with two simple examples that might be presented in a sophomore dynamic-systems course. The responses of these systems are calculated with both time-domain and Laplace transform techniques. We also use these examples to illustrate the advantages of the $\mathcal{L}_{-}$form. The section "A Brief Treatment of Generalized Functions," presents a theory of generalized functions incorporating nonzero pre-initial conditions. Using this brief theory, properties (4), (5) and some extensions are
$v_{I}(t)=\left\{\begin{array}{l}1 \mathrm{~V}, t<0 \\ 0 \mathrm{~V}, t>0\end{array}\right.$

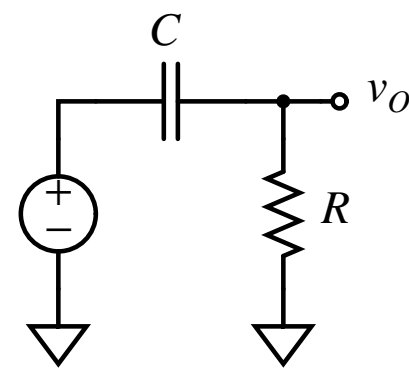

Fig. 1: Schematic of a highpass electrical filter driven by a step from 1 to 0 at $t=0$. The initial state of this system is the capacitor voltage $v_{C}\left(0^{-}\right)=1 \mathrm{~V}$, and thus the initial output voltage is $v_{O}\left(0^{-}\right)=0 \mathrm{~V}$. Although the unilateral Laplace transform of the input $v_{I}(t)$ is $V_{i}(s)=$ 0 , the presence of the nonzero pre-initial capacitor voltage produces a dynamic response.

developed more fully in the section "Generalized Functions and the Laplace Transform". Finally, we comment further on the treatment of the unilateral Laplace transform in the textbook literature.

\section{LAPLACE ANALYSIS EXAMPLES}

The need for careful definitions for the unilateral Laplace transform can perhaps be best appreciated by two simple examples. The first example is an RC circuit. The second example is a simplified model of an automobile suspension.

\section{A. Circuit Example}

Consider the highpass filter shown in Figure 1 driven by the voltage

$$
v_{I}(t)= \begin{cases}1 \mathrm{~V}, & t<0, \\ 0 \mathrm{~V}, & t>0 .\end{cases}
$$

We also specify the pre-initial condition $v_{O}\left(0^{-}\right)=0 \mathrm{~V}$, and thus the capacitor is initially charged to $v_{C}\left(0^{-}\right)=1 \mathrm{~V}$. This circuit is described by the differential equation

$$
C \frac{d}{d t}\left(v_{I}-v_{O}\right)-\frac{v_{O}}{R}=0
$$

or, equivalently,

$$
\frac{d v_{O}}{d t}+\frac{v_{O}}{R C}=\frac{d v_{I}}{d t}
$$

This example is solved by means of time-domain, $\mathcal{L}_{+}$, and $\mathcal{L}_{-}$ approaches to illuminate their advantages and disadvantages.

1) Physical Reasoning: An experienced analyst might approach the problem using the following time-domain argument: With the given input, the capacitor voltage is continuous at $t=0$ since there are no impulsive currents: that is, $v_{C}\left(0^{-}\right)=v_{C}\left(0^{+}\right)=1 \mathrm{~V}$. With the input step of $-1 \mathrm{~V}$, the output thus takes a step of $-1 \mathrm{~V}$ at $t=0$, and thus $v_{O}\left(0^{+}\right)=$ $-1 \mathrm{~V}$. Furthermore, in steady state with the input $v_{I}(t)$ equal to zero, the loop current is zero, and thus $\lim v_{O}(t)=0 \mathrm{~V}$. The system has a single time constant $\tau=\stackrel{t \rightarrow \infty}{R C}$; these initial and final values are satisfied by the homogeneous response $v_{O}(t)=-e^{-t / \tau}$ for $t>0$. This function is shown in Figure 2 along with the input waveform.

This approach has the advantage that it is physically based and doesn't require any signficant mathematical machinery. 

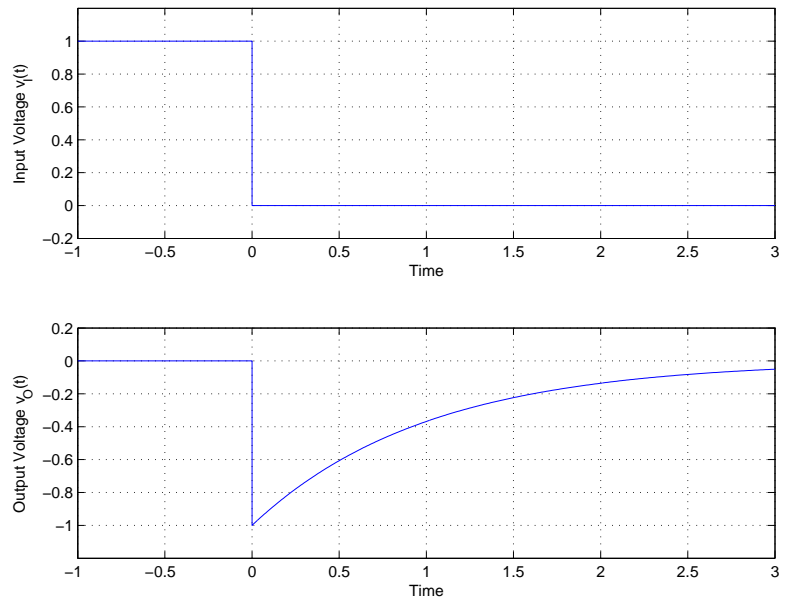

Fig. 2: Response of the highpass filter driven by a step from 1 to 0 at $t=0$, with $R C=1 \mathrm{~s}$. The presence of the nonzero pre-initial capacitor voltage produces the dynamic response shown here. Using the $\mathcal{L}_{-}$transform produces the correct result shown here without requiring a separate calculation of the transient at $t=0$.

However, such intuition is difficult to teach, particularly in an introductory course, where this approach appears to require feats of insight that are hard for students to generalize.

2) The $\mathcal{L}_{+}$Laplace Transform Approach: The circuit problem can be solved systematically using the Laplace transform. Using the $\mathcal{L}_{+}$version of the transform (1), we proceed as follows: The Laplace transform of the differential equation (6), using the time-derivative rule (2), is

$$
s V_{o}(s)-v_{O}\left(0^{+}\right)+\frac{V_{o}(s)}{R C}=s V_{i}(s)-v_{I}\left(0^{+}\right),
$$

which yields

$$
V_{o}(s)=\frac{s V_{i}(s)-v_{I}\left(0^{+}\right)+v_{O}\left(0^{+}\right)}{s+1 / \tau} .
$$

The transform of the input is

$$
\mathcal{L}_{+}\left\{v_{I}(t)\right\}=V_{i}(s)=0,
$$

which certainly seems useless; however, we have not yet considered the effect of initial conditions.

Unfortunately, the $\mathcal{L}_{+}$approach requires the values of signals at $t=0^{+}$. Since the initial conditions are given naturally at $t=0^{-}$, the post-inital values at $0^{+}$need to be determined so that the Laplace results can be calculated. In fact, determination of the $0^{+}$values requires additional effort, which depends either on some physical insight or the technique of impulse matching [29]. The $0^{+}$values are determined based on physical reasoning in the time-domain solution presented above.

Impulse matching is based on the insight that a differential equation must maintain equality at all times, and in particular must be balanced in all orders of singularity functions. For the first-order differential equation (6), with the input taking a step of $-1 \mathrm{~V}$ at $t=0$, the input term is $d v_{I} / d t=-\delta(t)$. To balance this term, the output must then contain a step of $-1 \mathrm{~V}$ at $t=0$ so that the $d v_{O} / d t$ term yields a matching $-\delta(t)$. We thus recognize that $v_{O}\left(0^{+}\right)=-1 \mathrm{~V}$. Since the required reasoning becomes increasingly convoluted as the system order grows, this approach is even harder to teach than one based on physical insight. An example of this approach for a second-order system is given in [29], pp. 342-3.

In any case, we now have the post-inital value $v_{O}\left(0^{+}\right)=$ $-1 \mathrm{~V}$. Furthermore, with the input step from 1 to $0, v_{I}\left(0^{+}\right)=$ $0 \mathrm{~V}$. Using these results, we obtain

$$
V_{o}(s)=\frac{-1}{s+1 / \tau}
$$

and

$$
v_{O}(t)=-e^{-t / \tau}, \quad t>0,
$$

which is the same result derived earlier.

3) The $\mathcal{L}_{-}$Approach: The circuit solution using the $\mathcal{L}_{-}$ version of the transform (3) proceeds as follows: The Laplace transform of the differential equation (6), using the timederivative rule (4), yields

$$
V_{o}(s)=\frac{s V_{i}(s)-v_{I}\left(0^{-}\right)+v_{O}\left(0^{-}\right)}{s+1 / \tau} .
$$

The associated initial values are $v_{I}\left(0^{-}\right)=1 \mathrm{~V}$ and $v_{O}\left(0^{-}\right)=$ $0 \mathrm{~V}$.

With $V_{i}(s)=0$ and $v_{O}\left(0^{-}\right)=0 \mathrm{~V}$ the expression (8) simplifies to

$$
V_{o}(s)=\frac{-1}{s+1 / \tau} .
$$

Inverting this transform gives the output waveform

$$
v_{O}(t)=-e^{-t / \tau}, \quad t>0,
$$

as before. We can further apply the initial-value theorem to (9), calculating

$$
v_{O}\left(0^{+}\right)=\lim _{s \rightarrow \infty \cdot 1} s V_{o}(s)=-1 .
$$

The $\mathcal{L}_{-}$Laplace transform solution has the advantage that it is general; students can apply this approach to any linear constant-coefficient differential equation. This technique can be readily taught and used by students at the introductory level, and yields correct results in the presence of nonzero pre-initial values or input functions containing singularities.

Of course, both forms of the Laplace transform yield correct results. However, the $\mathcal{L}_{+}$approach requires the additional step of finding $v_{O}\left(0^{+}\right)$, which is not required with the $\mathcal{L}_{-}$ form of the transform. This disadvantage is true in general. Using the $\mathcal{L}_{-}$transform (3) and the properties (4) and (5), all singularity functions at $t=0$ are included in the domain of analysis, yielding parallel advantages over the $\mathcal{L}_{+}$form. Furthermore, students attempting to use the $\mathcal{L}_{+}$form should be rightly puzzled by the null identity $\mathcal{L}_{+}\{\delta(t)\}=0$, whereas $\mathcal{L}_{-}\{\delta(t)\}=1$.

\section{B. Automobile Suspension Example}

Another example that illuminates the use of the $\mathcal{L}_{-}$Laplace transform with pre-initial conditions is the idealized secondorder automobile-suspension system shown in Figure 3. In this simplified model, we assume that the center of the wheel follows the step contour exactly, such that the input motion $x(t)$ takes the form of a unit step. We are then 


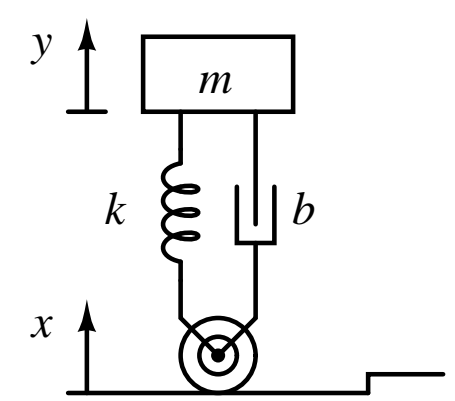

Fig. 3: An idealized automobile-suspension system approaching a rectangular speed bump. In the analysis, the center of the wheel is assumed to follow the bump exactly. Analyzing the effect of this input in the presence of an arbitrary initial state illustrates the advantages of using the $\mathcal{L}_{-}$form of the Laplace transform.

interested in calculating the resulting car-body motion $y(t)$. We examine this response under three sets of initial conditions to provide insight into the types of solutions that might be calculated using Laplace techniques. The alternative approach using the $\mathcal{L}_{+}$form is more cumbersome here than in the firstorder case above, and so is not discussed further.

The differential equation describing the system in Figure 3 is

$$
m \ddot{y}=b(\dot{x}-\dot{y})+k(x-y),
$$

where $m$ is the mass of the car, $b$ is the damping coefficient of the suspension, and $k$ is the spring constant of the suspension. The Laplace transforms of the derivatives are

$$
\begin{aligned}
& \mathcal{L}_{-}\{\dot{x}\}=s X(s)-x\left(0^{-}\right), \\
& \mathcal{L}_{-}\{\dot{y}\}=s Y(s)-y\left(0^{-}\right),
\end{aligned}
$$

and

$$
\mathcal{L}_{-}\{\ddot{y}\}=s^{2} Y(s)-s y\left(0^{-}\right)-\dot{y}\left(0^{-}\right) .
$$

Therefore, the differential equation transforms to

$$
\begin{aligned}
& m s^{2} Y(s)-m s y\left(0^{-}\right)-m \dot{y}\left(0^{-}\right)= \\
& \quad b\left(s X(s)-x\left(0^{-}\right)-s Y(s)+y\left(0^{-}\right)\right)+k(X(s)-Y(s)) .
\end{aligned}
$$

Solving for the output $Y(s)$ gives

$$
Y(s)=\frac{(b s+k) X(s)}{m s^{2}+b s+k}+\frac{-b x\left(0^{-}\right)+(m s+b) y\left(0^{-}\right)+m \dot{y}\left(0^{-}\right)}{m s^{2}+b s+k}
$$

Using this solution, we can readily find the system response to arbitrary Laplace-transformable inputs and general initial conditions.

We now assume that the input takes the form of a one-meter step $x(t)=u(t)$, which has the transform $X(s)=1 / s$ and a pre-initial value $x\left(0^{-}\right)=0 \mathrm{~m}$. The solution under this step is then calculated for three sets of initial conditions. The first set of initial conditions for the output and its derivative are the initial-rest conditions $y\left(0^{-}\right)=0 \mathrm{~m}$ and $\dot{y}\left(0^{-}\right)=0 \mathrm{~m} / \mathrm{s}$. From (10) the resulting output transform is

$$
Y(s)=\frac{b s+k}{s\left(m s^{2}+b s+k\right)} .
$$

Inverting $Y(s)$ yields the zero-state step response shown in Figure 4(a). Note that in this system, for a step input, the
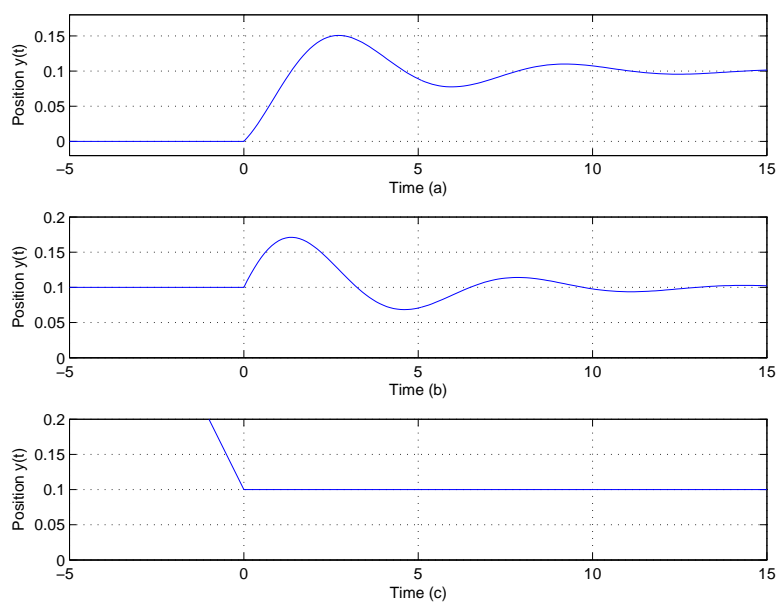

Fig. 4: Response of the idealized suspension system (plotted for $m=1000 \mathrm{~kg}, b=500 \mathrm{~N}-\mathrm{s} / \mathrm{m}$, and $k=1000 \mathrm{~N} / \mathrm{m}$ ) to an input unit step with three sets of initial conditions: (a) initial rest; (b) initial position $y\left(0^{-}\right)=1 \mathrm{~m}$, zero initial velocity $\dot{y}\left(0^{-}\right)=0 \mathrm{~m} / \mathrm{s}$; and (c) initial position $y\left(0^{-}\right)=1 \mathrm{~m}$ and initial velocity $\dot{y}\left(0^{-}\right)=-b / m$. The last set of initial conditions results in immediate convergence to final value.

position is continuous across $t=0$, while the velocity takes a step with value $b / \mathrm{m}$. These actions occur independently of initial conditions and thus apply to all three cases studied here.

The second set of initial conditions consists of the initial position $y\left(0^{-}\right)=1 \mathrm{~m}$ with zero initial velocity $\dot{y}\left(0^{-}\right)=$ $0 \mathrm{~m} / \mathrm{s}$. That is, the system position starts at the level to which it settles in steady state. In this case, the output transform expression from (10) is

$$
Y(s)=\frac{1}{s}+\frac{b}{m s^{2}+b s+k} .
$$

Inverting this expression gives the time response shown in Figure 4(b). The observed motion results from the force impulse $b \delta(t)$ that the damper applies to the mass at the moment of the step. Applying the initial-value theorem to $Y(s)$ and $s Y(s)$, respectively, yields the post-initial values $y\left(0^{+}\right)=$ $1 \mathrm{~m}$ and $\dot{y}\left(0^{+}\right)=b / m$. The post-initial velocity is simply an expression of the change in momentum induced in the mass by the force impulse applied from the damper; this change in momentum occurs in all three cases studied here.

The third set of initial conditions is selected to yield immediate convergence to the final value of the response. That is, we choose the initial position as $y\left(0^{-}\right)=1 \mathrm{~m}$ and set the initial velocity to $\dot{y}\left(0^{-}\right)=-b / m$, such that the initial velocity is exactly canceled by the force impulse from the damper. For these initial conditions, the output transform (10) is

$$
Y(s)=\frac{m s^{2}+b s+k}{s\left(m s^{2}+b s+k\right)}=\frac{1}{s} .
$$

We recognize this result as the transform of the unit step, which gives

$$
y(t)=1 \mathrm{~m}, \quad t>0 .
$$

This waveform is shown in Figure 4(c), along with a pre-initial velocity of $-b / m$ as indicated by the straight line segment of position for $t<0$. Of course, in the analysis we do not concern ourselves with exactly how this pre-initial velocity is 
established, nor any details of the time variation prior to the transient. The only required prior knowledge is the velocity and position immediately before the transient at $t=0^{-}$.

\section{A BRIEF TREATMENT OF GenERALIZED FunCtions}

Many engineering and mathematics textbook discussions of the unilateral Laplace transform treat singularities at $t=0$ differently from singularities at $t>0$. These inconsistencies arise from a reluctance to use the generalized derivative. However, a consistent and easily understood treatment can be based on the uniform use of generalized functions [30]. In this section, we provide an accessible mathematical account of a portion of the theory of generalized functions that is broad enough for most engineering applications and free of mathematical technicalities.

The treatment of generalized functions presented below is just a small part of the standard mathematical theory of distributions, but what we present is both sufficiently concrete to be quickly understood, and sufficiently general to be widely useful. Our treatment is axiomatic in the sense that the delta function and its derivatives are treated purely symbolically. We describe the behavior of singularity functions in a way that we believe supports the common intuition about them.

Because of our focus on the unilateral Laplace transform, we consider generalized functions on the interval $[0, \infty)$. On the other hand, the applications presented above indicate the desirability of incorporating nonzero pre-initial conditions, and we build this possibility into the theory by incorporating into the information carried by a generalized function the pre-initial values at $t=0^{-}$of the function and all of its derivatives. We note, however, that this choice is not wellsuited for a discussion of convolution. Restricting to rest preinitial conditions $f^{(n)}\left(0^{-}\right)=0$, without further changes, results in a satisfactory treatment of convolution and has other advantages as well, but loses the flexibility of non-rest preinitial conditions. Both theories are useful.

\section{A. Definitions}

1) Sparsity: A set $S$ of numbers is sparse if every interval of finite length contains only finitely many elements of $S$.

2) Piecewise continuity: A function $f(t)$ on $[0, \infty)$ is piecewise continuous if there is a sparse set $S \subset[0, \infty)$ such that $f(t)$ is continuous on the complement of $S$ and, for every $a \in[0, \infty)$, the one-sided limits $f\left(a^{+}\right)$and $f\left(a^{-}\right)$exist.

The expression $f\left(0^{-}\right)$requires special interpretation, since the usual meaning given to the one-sided limit, namely,

$$
f\left(a^{-}\right)=\lim _{t \uparrow a} f(t),
$$

is unavailable to us because $f(t)$ is defined only for $t>0$. Rather, the value $f\left(0^{-}\right)$represents additional information that must be specified to completely specify the function. This information is a pre-initial condition.

3) Piecewise smoothness: A function $f(t)$ defined on the interval $[0, \infty)$ is piecewise smooth if there is a sparse set $S \subset[0, \infty)$ such that all of the derivatives of $f(t)$ exist on the complement of $S$ and have left and right limits at 0 and at every element of $S$. Thus, part of the data of a piecewisesmooth function on $[0, \infty)$ is the sequence of values $f^{(n)}\left(0^{-}\right)$, for $n=0,1,2 \ldots$. These data are not implied by values of $f(t)$ for $t \geq 0$. In fact, the series formed using these values,

$$
\sum_{n=0}^{\infty} \frac{f^{(n)}\left(0^{-}\right)}{n !} x^{n},
$$

may not be the Maclaurin series of any function; it may have radius of convergence zero. Normally, however, these values are given as the limiting values of a function defined for $t<0$.

In this setting, the unit-step function $u(t)$ is defined to take on the value 1 for all $t>0$ and have zero pre-initial conditions $u^{(n)}\left(0^{-}\right)=0$ for all $n \geq 0$. The value of a generalized function at any single point is not significant, as explained below.

4) Singularity functions: A singularity function is a formal sum of the form

$$
f_{s}(t)=\sum_{k, l} c_{k, l} \delta^{(l)}\left(t-a_{k}\right),
$$

where $a_{k}$ is a nondecreasing sequence in $[0, \infty)$. For each value of $k$, only finitely many of the $c_{k, l}$ are allowed to be nonzero. The sum can be over a finite or infinite range of values of $k$. If the range of values of $k$ is infinite, we require that $\lim a_{k}=\infty$. In this treatment, the symbol $\delta$ is used symbolically. A virtue of this axiomatic approach is that it implements pre-existing intuitions the user may have without introducing distracting artifices. Of course, $\delta(t)$ refers to the unit impulse, and $\delta^{(l)}$ is its $l^{\text {th }}$ derivative. In particular, $\delta^{(0)}(t)=\delta(t)$.

The constants $a_{k}$ for which some $c_{k, l}$ is nonzero are the singular points of $f_{s}(t)$. The singularity at $t=a$ of $f_{s}(t)$ is the part of the sum corresponding to $a_{k}=a$. If this sum is empty, $f_{s}(t)$ is nonsingular at $t=a$.

We comment here that the unit-step function $u(t)=$ $\delta^{(-1)}(t)$ is taken as dimensionless. Succeeding derivatives introduce units of increasing negative powers of time. The units of the singularity function $\delta^{(l)}(t)$ are thus seconds ${ }^{-(l+1)}$. If we wish to associate these functions with physical quantities, then the functions must be multiplied by weighting terms with appropriate units. For example, in a voltage waveform, the functions $\delta^{(l)}(t)$ must be multiplied by weighting terms $c_{k, l}$ that have units of $\mathrm{V}-\mathrm{s}{ }^{(l+1)}$. As another example, a force impulse $F_{0} \delta(t)$ has a weighting term $F_{0}$ with units of N-s. The term $F_{0}$ then corresponds to the area under a finite-duration force event, such as a hammer blow, which we might model with an impulse function.

5) Generalized functions: A generalized function is a formal expression

$$
f(t)=f_{r}(t)+f_{s}(t),
$$

whose regular part $f_{r}(t)$ is determined by a piecewise-smooth function and whose singular part $f_{s}(t)$ is a singularity function. The regular part contributes the pre-initial conditions $f^{(n)}\left(0^{-}\right)=f_{r}^{(n)}\left(0^{-}\right)$.

There is a subtlety here. Two piecewise-smooth functions define the same generalized functions if and only if they differ from each other on a sparse set. This fact is why the value of 
the unit-step function at $t=0$ is irrelevant. Consequently, the values attained on any sparse set are irrelevant to the identity of the corresponding generalized function. When viewing a function as a generalized function, it is best never to refer to specific values $f(a)$, which are always irrelevant, but rather to the one-sided limits $f\left(a^{+}\right)$and $f\left(a^{-}\right)$, which are always defined (even for $a \in S$ ), even if a finite number of values of $f(t)$ are changed. This caveat is necessary to ensure that the standard uniqueness theorem for solutions of differential equations holds for generalized functions.

6) Generalized differentiation: Generalized functions can be differentiated to give additional generalized functions. To begin with, let $f(t)$ be a piecewise-smooth function that is smooth on the complement of a sparse set $S$. The generalized derivative of a piecewise-smooth function $f(t)$ is a generalized function that is denoted by the standard notation $f^{\prime}(t)$ for the derivative. The regular part of $f^{\prime}(t)$ is smooth on the complement of $S$, and is (by definition, in this treatment) given by the ordinary derivative of $f_{r}(t)$ on the complement of $S$. The pre-initial values are specified by $\left(f^{\prime}\right)^{(n)}\left(0^{-}\right)=$ $f_{r}^{(n+1)}\left(0^{-}\right)$. The singular part of $f^{\prime}(t)$ is given by summing delta functions that arise from the jumps in the graph of $f(t)$, that is,

$$
f_{s}^{\prime}(t)=\sum_{a \in S}\left(f\left(a^{+}\right)-f\left(a^{-}\right)\right) \delta(t-a) .
$$

The generalized derivative of a singularity function simply follows the notation

$$
\left(\delta^{(l)}\right)^{\prime}(t-a)=\delta^{(l+1)}(t-a) .
$$

\section{B. Properties of Generalized Functions}

1) Multiplication: Generalized functions cannot necessarily be multiplied together, and such products never occur in applications of the Laplace transform. However, if $g(t)$ is a smooth function then its product with any generalized function $f(t)$ is defined, and the product rule

$$
(f \cdot g)^{\prime}(t)=f^{\prime}(t) g(t)+f(t) g^{\prime}(t)
$$

holds in this generality. The following paragraphs re-express this product in the standard form we use to define generalized functions.

Since we want the product (11) to be linear in each variable, and of course we want the product of $f_{r}(t) g(t)$ to be the usual product of functions, it suffices to define $f(t) g(t)$ for $g(t)$ smooth and $f(t)=\delta^{(n)}(t-a)$. To decide how to define $g(t) \delta^{(n)}(t-a)$ for $g(t)$ smooth, we begin with the case $n=0$, and simply define the product $g(t) \delta(t-a)$ to be what we expect it to be, namely,

$$
g(t) \delta(t-a)=g(a) \delta(t-a) .
$$

It turns out that (12), along with the product rule (11), determines expressions for all products of the form $g(t) \delta^{(n)}(t-a)$, where $g(t)$ is a smooth function, as linear combinations of terms of the form $\delta^{(n-k)}(t-a)$ with constant coefficients. To see this result, we differentiate (12) and apply this same identity with $g^{\prime}(t)$ replacing $g(t)$ to find

$$
g(t) \delta^{\prime}(t-a)=g(a) \delta^{\prime}(t-a)-g^{\prime}(a) \delta(t-a) .
$$

Continuing in the same way leads by induction to the identity

$$
g(t) \delta^{(n)}(t-a)=\sum_{k=0}^{n}(-1)^{k}\left(\begin{array}{l}
n \\
k
\end{array}\right) g^{(k)}(a) \delta^{(n-k)}(t-a) .
$$

This formula defines how a generalized function is to be multiplied by a smooth function $g(t)$. In addition, (13) explains how smooth $g(t)$ must be so that the product $g(t) \delta^{(n)}(t-a)$ makes sense, specifically, $g(t)$ and its derivatives up to $g^{(n)}(t)$ must exist and be continuous at $t=a$. This perspective is taken by [31]; see Equation 3.26.

Having arrived at a definition of the product of a smooth function with a generalized function, we now check that the product rule (11) does in fact hold. The product rule can be integrated to obtain the usual integration-by-parts formula, valid for any smooth function $g(t)$ and any generalized function $f(t)$, given by

$$
\left.\int_{a^{-}}^{b^{+}} f^{\prime}(t) g(t) d t=f(t) g(t)\right]_{a^{-}}^{b^{+}}-\int_{a^{-}}^{b^{+}} f(t) g^{\prime}(t) d t .
$$

To apply (14) to the case $a=0$, we use the values $f\left(0^{-}\right)$and $g\left(0^{-}\right)$that are included in the definitions of these functions. It follows from the definition of smoothness (as opposed to piecewise smoothness) that $g\left(0^{-}\right)=g(0)$. Hence

$\int_{0^{-}}^{b^{+}} f^{\prime}(t) g(t) d t=\left(f\left(b^{+}\right) g(b)-f\left(0^{-}\right) g(0)\right)-\int_{0^{-}}^{b^{+}} f(t) g^{\prime}(t) d t$.

2) Convolution: The convolution product cannot be defined on the whole of our space of generalized functions so as to have expected properties. Convolution with $\delta(t-a)$, where $a \geq 0$, should be a shift, that is

$$
\delta(t-a) * f(t)=\left\{\begin{array}{l}
f(t-a) \text { if } t>a, \\
0 \text { if } t<a .
\end{array}\right.
$$

When $a>0$, the right-hand side determines the values of the convolution and its derivative at $t=a^{-}$, independently of the values $f^{(n)}\left(0^{-}\right)$; these values are not shifted, but rather are simply lost.

The best option is to define the convolution product only on the subspace of generalized functions with rest pre-initial conditions. Equation (16) together with

$$
\delta^{(n)}(t) * f(t)=f^{(n)}(t)
$$

which is valid for all generalized functions $f(t)$, and the assertion of associativity and commutativity define the convolution product on this subspace. Note that (17) for $f(t)=\delta^{(m)}(t)$ gives the equation $\delta^{(n)}(t) * \delta^{(m)}(t)=\delta^{(n+m)}(t)$. In particular, $\delta(t) * \delta(t)=\delta(t)$

\section{Generalized Functions And THE LAPLACE TRANSFORM}

Laplace transforms can be defined for generalized functions satisfying appropriate exponential growth conditions. The basic singularity functions satisfy

$$
\mathcal{L}_{-}\left\{\delta^{(n)}(t)\right\}=s^{n},
$$


which we take as a definition. Specifying these values is all that is required to develop a useful theory of the Laplace transform of generalized functions, as shown below.

Convergence of the improper integral defining the Laplace transform requires conditions on both regular and singular functions. One requirement for a piecewise-continuous function $f_{r}(t)$ is that it be of exponential type, meaning that there exists a constant $M$ such that $\left|f_{r}(t)\right|<e^{M t}$ for all large $t$. Then the integral converges for $\Re \mathrm{e}(s)>M$.

A convenient and sufficiently general condition on singularity functions that guarantees convergence of the Laplace transform in a right-half plane is given as follows. First, we require that the singularity function is of finite order, meaning that there is a largest $n$ for which $\delta^{(n)}(t-a)$ occurs. Then, in the expression

$$
f_{s}(t)=\sum_{k, l} c_{k, l} \delta^{(l)}\left(t-a_{k}\right),
$$

we require that $f_{s}(t)$ also be of exponential type, meaning that there exists a constant $N$ such that $\left|c_{k, l}\right|<e^{N a_{k}}$ for all large $k$ and for all $l$. With these assumptions, the sum defining $\mathcal{L}_{-}\left\{f_{s}(t)\right\}$ converges for all $\Re \mathrm{e}(s)>N$.

For a generalized function $f(t)=f_{r}(t)+f_{s}(t)$, we require that the regular part $f_{r}(t)$ be of exponential type and that the singular part $f_{s}(t)$ be of finite order and of exponential type.

\section{A. The Time-Derivative Rule}

The time-derivative rule (4) can be directly derived from the definition (3) when applied to $f^{\prime}(t)$, that is,

$$
\mathcal{L}_{-}\left\{f^{\prime}(t)\right\}=\int_{0^{-}}^{\infty} f^{\prime}(t) e^{-s t} d t .
$$

Integrating by parts following (15)

$\int_{0^{-}}^{\infty} f^{\prime}(t) g(t) d t=f(\infty) g(\infty)-f\left(0^{-}\right) g(0)-\int_{0^{-}}^{\infty} f(t) g^{\prime}(t) d t$

with

$$
g(t)=e^{-s t}
$$

and

$$
g^{\prime}(t)=-s e^{-s t}
$$

yields

$$
\begin{aligned}
\int_{0^{-}}^{\infty} f^{\prime}(t) e^{-s t} d t & =-f\left(0^{-}\right) e^{0}+\int_{0^{-}}^{\infty} f(t) s e^{-s t} d t \\
& =-f\left(0^{-}\right)+s \mathcal{L}_{-}\{f(t)\} .
\end{aligned}
$$

With systematic use of the generalized derivative, this formula is valid for any generalized function $f(t)$ of exponential type provided that $s$ has real part sufficiently large for the improper integrals to converge.

\section{B. The Initial-Value Theorem}

The initial-value theorem (5) is perhaps more accurately called the post-initial-value theorem, since it yields the result at $0^{+}$. However, since there is no "pre-initial-value theorem," we retain standard terminology and refer to (5) as the initial-value theorem. This result can be derived by several approaches that provide alternative insights. We show two approaches below, which are based upon a formal application of the derivative rule and the initial-singularity theorem. In addition, a qualitative argument based on the sifting property of $s e^{-s t}$ can be found in [10], p. 12.

The initial-value theorem extends to the situation in which $F(s)$ fails to converge to zero as $s \rightarrow \infty \cdot 1$, but can be written as

$$
F(s)=\tilde{F}(s)+\sum_{n=1}^{N} a_{n} s^{n},
$$

where the function $\tilde{F}(s)$ converges to zero in the limit $s \rightarrow$ $\infty \cdot 1$. In this case, the post-initial value of $f$ is given by

$$
\lim _{s \rightarrow \infty \cdot 1} s \tilde{F}(s)=f\left(0^{+}\right) .
$$

Said another way, the occurence of singularity functions in $f(t)$ does not preclude a well-defined limit at $t=0^{+}$, and the value of this limit is captured by the Laplace transform.

1) From the Derivative Rule: The initial-value theorem can be derived from the derivative rule (4), which implies

$$
s F(s)=\int_{0^{-}}^{\infty} f^{\prime}(t) e^{-s t} d t+f\left(0^{-}\right) .
$$

Taking the limit as $s$ goes to infinity along the real axis gives

$$
\begin{aligned}
\lim _{s \rightarrow \infty \cdot 1} s F(s) & =\lim _{s \rightarrow \infty \cdot 1}\left(\int_{0^{-}}^{\infty} f^{\prime}(t) e^{-s t} d t\right)+f\left(0^{-}\right) \\
& =\lim _{s \rightarrow \infty \cdot 1}\left(\int_{0^{-}}^{0^{+}} f^{\prime}(t) e^{0} d t+\int_{0^{+}}^{\infty} f^{\prime}(t) e^{-s t} d t\right)+f\left(0^{-}\right. \\
& \left.=\lim _{s \rightarrow \infty \cdot 1}(f(t)]_{0^{-}}^{0^{+}}+0\right)+f\left(0^{-}\right) \\
& =f\left(0^{+}\right)
\end{aligned}
$$

which is the expected result.

2) From the Initial-Singularity Formula: The initial-value theorem can perhaps best be thought of as a special case of what we call the initial-singularity theorem, which asserts that $F(s)$ is asymptotic, as $s$ increases through the real numbers, to a polynomial that carries information about the singularity of $f(t)$ at $t=0$. To express this relation we use the notation $F(s) \sim G(s)$ to represent

$$
\lim _{s \rightarrow \infty \cdot 1}(F(s)-G(s))=0 .
$$

The initial-singularity formula asserts that if the singularity of $f(t)$ at $t=0$ is

$$
\sum_{l} c_{l} \delta^{(l)}(t)
$$

then, with $F(s)=\mathcal{L}_{-}\{f(t)\}$,

$$
F(s) \sim \sum_{l} c_{l} s^{l} .
$$

This result comes out of the relation $\mathcal{L}_{-}\left\{\delta^{(n)}(t)\right\}=s^{n}$ together with the two facts

$$
\lim _{s \rightarrow \infty \cdot 1} \mathcal{L}_{-}\left\{f_{r}(t)\right\}=0
$$


and, for all $a>0$,

$$
\lim _{s \rightarrow \infty \cdot 1} \mathcal{L}_{-}\left\{\delta^{(n)}(t-a)\right\}=0 .
$$

The initial-value theorem arises by applying the initialsingularity theorem to $f^{\prime}(t)$. The singularity of $f^{\prime}(t)$ at $t=0$ is

$$
\left(f\left(0^{+}\right)-f\left(0^{-}\right)\right) \delta(t)+\sum_{l} c_{l} \delta^{(l+1)}(t),
$$

with Laplace transform

$$
f\left(0^{+}\right)-f\left(0^{-}\right)+\sum_{l} c_{l} s^{l+1} .
$$

Hence the time-derivative rule (4) implies

$s F(s)-f\left(0^{-}\right)=\mathcal{L}_{-}\left\{f^{\prime}(t)\right\} \sim f\left(0^{+}\right)-f\left(0^{-}\right)+\sum_{l} c_{l} s^{l+1}$.

Canceling the $f\left(0^{-}\right)$terms yields

$$
s F(s) \sim f\left(0^{+}\right)+\sum_{l} c_{l} s^{l+1} .
$$

In particular, if $f(t)$ is nonsingular at $t=0$, then

$$
\lim _{s \rightarrow \infty \cdot 1} s F(s)=f\left(0^{+}\right) .
$$

More generally, with the above analysis, we can see that the value $f\left(0^{+}\right)$can be read off directly from an expression for the Laplace transform. That is, if $F(s)$ is a polynomial plus a function $\tilde{F}(s)$ converging to zero as $s \rightarrow \infty \cdot 1$, then

$$
\lim _{s \rightarrow \infty \cdot 1} s \tilde{F}(s)=f\left(0^{+}\right) .
$$

For example, consider the first-order transform

$$
F(s)=\frac{s+a}{s+b}=1+\frac{a-b}{s+b} .
$$

This function has a post-initial value $f\left(0^{+}\right)=a-b$, even though $f(t)$ includes an impulse at $t=0$, as can be seen using our result above, or through inversion of the transform.

\section{Comments on the TeXtbook Literature}

The textbook literature is surprisingly random in its treatment of the origin in the unilateral Laplace transform; many otherwise-excellent texts fumble this issue. We found ourselves confused by various textbook accounts, and our experiences provided the initial impetus for writing this article.

Textbooks that use

$$
\mathcal{L}\left\{f^{\prime}(t)\right\}=s F(s)-f(0)
$$

or

$$
\lim _{s \rightarrow \infty} s F(s)=f(0)
$$

without further clarification must be considered suspect. These expressions appear in many texts on differential equations [32]-[36] and as well as control books such as [37]-[40]. The control book [41] uses 0 in these expressions, but then inexplicably switches to $0^{+}$when transforming the unit step and $0^{-}$when transforming the unit impulse. The first three editions of [42] use 0 everywhere. In the fourth [43] and later editions, the author correctly states that "strictly" the transform "should be defined from $t=0^{-}$," but then incorrectly reasons that the "fine point of using $0^{-}$or $0^{+}$never needs to be addressed," and continues to use 0 everywhere. The most recent edition [44] does not clarify this issue.

As discussed earlier, the $\mathcal{L}_{+}$form of the transform has the critical disadvantage that it leaves transients at $t=0$ as essentially an exercise for the reader (or worse yet, the student). Some books such as [45] and [46] add to the confusion by showing both $\mathcal{L}_{+}$and $\mathcal{L}_{-}$forms, with little or no comment as to when to choose between them. These books also say that the $\mathcal{L}_{+}$form must be used to derive the initialvalue theorem, which, as shown above, is false. The systems book [47] states that using the one-sided transform is the same as assuming that signals are zero for $t<0$, which is not true, as can be seen in the context of the examples in this article. The book [25] states that both forms are acceptable as long as the corresponding properties are developed in a consistent fashion. However, this approach leads to some convoluted reasoning associated with the derivative property and with the transform of the unit impulse.

The first two editions of [48] use $0^{-}$in the lower limit on the Laplace integral and in the derivative property. However, the statement of the initial-value theorem in [48] notifies the reader that "in general, the values $y\left(0^{-}\right), y(0)$, and $y\left(0^{+}\right)$ for a Laplace-transformable function can differ," but does not specify which value is calculated by the initial-value theorem. In the third and fourth editions [49] the initial-value theorem is given incorrectly as $\lim _{s \rightarrow \infty} s Y(s)=y\left(0^{-}\right)$.

The first edition of [50] uses the $\mathcal{L}_{+}$convention and includes an example where the initial conditions are conveniently but unrealistically given in terms of $y\left(0^{+}\right)$. The sixth edition still uses the $\mathcal{L}_{+}$convention, but the $\mathcal{L}_{-}$convention is used in the seventh [51] and later editions (although without comment on impulses at $t=0$ or even a discussion of the initial-value theorem). The first edition of the popular signals and systems book [52] briefly covers the $\mathcal{L}_{+}$form of the the unilateral Laplace transform, while the second edition [53] includes a much more thorough discussion using the $\mathcal{L}_{-}$transform and an example with $\delta(t)$. While the first edition of [54] uses $0^{-}$ in the lower limit on the Laplace integral, the $f(0)$ term in the derivative property is unclear. The third [55] and subsequent editions contain a clear and complete discussion of the $\mathcal{L}_{-}$ convention, including the derivative property (4), explaining, "It is the transform of the unit impulse that lead us to choose the $\mathcal{L}_{-}$transform rather than the $\mathcal{L}_{+}$transform."

A rigorous foundation for the $\mathcal{L}_{-}$convention of the Laplace transform requires a supporting treatment of singularity functions. The sidebar "Distinguishing $0,0^{+}$, and $0^{-}$" discusses the application of the Laplace transform to abstract signals, independent of any dynamic-systems context, to clarify the need for consistent definitions of derivatives and the Laplace transform. Recently, some authors [56], [57] have suggested that the theory of generalized functions is far too complicated for use in control education. We believe the treatment of generalized functions presented in the previous section is brief enough to be quickly understood, yet sufficiently general to be widely useful. Reference [57] further suggests that 
singularities such as $\delta(t)$ must be avoided in undergraduate teaching, and introduces a somewhat convoluted procedure for handling derivatives of discontinuous functions within this unusual perspective. This approach is simply not practical, either at the elementary or advanced levels, for teaching or research.

Remarkably, the first control text to explicitly and consistently use the $\mathcal{L}_{-}$form is also one of the first control books, the landmark classic text [58]. Starting on page 51 is a complete discussion of the definition of the $\mathcal{L}_{-}$transform, the correct derivative rule, the initial-value theorem, and a simple statement of the initial-singularity theorem (all using Heaviside's $p$ instead of $s$ as the Laplace variable, as is common in older texts). Notably, [58] does not reference the misleading [18].

\section{CONCLUSION}

While all of the textbooks referenced above have much to recommend them, the issue of Laplace techniques requires clarification in many of these references, as well as in the teaching of systems. It is important to introduce a treatment of the unilateral Laplace transform that includes transients at the origin. As made clear in the above discussions, we regard the $\mathcal{L}_{-}$form as the best approach for studying dynamic systems, since it easily incorporates nonzero pre-initial conditions and inputs that include singularity functions. This form is adopted and correctly treated in many texts, such as [8]-[12], [29], [51], [53], [55], and [58]. We also note that the trend is toward the increased and explicit use of $\mathcal{L}_{-}$in the revisions of many books, for example, from [50] to [51], from [52] to [53], and from [54] to [55]. We hope that the discussion in this article helps to convince controls educators to continue this trend.

In summary, for a set of properties for the unilateral Laplace transform that are useful to engineers, we advocate uniform use of the definition

$$
\mathcal{L}\{f(t)\}=\int_{0^{-}}^{\infty} f(t) e^{-s t} d t,
$$

the derivative rule involving pre-initial conditions

$$
\mathcal{L}\left\{f^{\prime}(t)\right\}=s F(s)-f\left(0^{-}\right),
$$

and the initial-value theorem

$$
\lim _{s \rightarrow \infty \cdot 1} s F(s)=f\left(0^{+}\right) .
$$

These properties give students general tools that can be used to solve differential equations with both nonzero initial conditions and inputs. These properties also work correctly in the presence of input singularities and non-rest pre-initial conditions. We find other presentations of the unilateral transform to be more difficult to apply and often confusing.

\section{REFERENCES}

[1] R. V. Churchill, Operational Mathematics. New York: McGraw-Hill, 1958.

[2] M. R. Spiegel, Schaum's Outline of Theory and Problems of Laplace Transforms. New York: Schaum Publishing, 1965.

[3] J. L. Melsa and D. G. Schultz, Linear Control Systems. New York: McGraw-Hill, 1969
[4] J. Van de Vegte, Feedback Control Systems, 3rd ed. Englewood Cliffs: Prentice Hall, 1994

[5] R. N. Bateson, Introduction to Control System Technology, 6th ed. Upper Saddle River: Prentice Hall, 1999.

[6] G. Doetsch, Theorie und Anwendung der Laplace-Transformation. New York: Dover, 1943.

[7] A. H. Zemanian, Distribution Theory and Transform Analysis. New York: McGraw-Hill, 1965.

[8] L. A. Zadeh and C. A. Desoer, Linear System Theory. New York: McGraw-Hill, 1963.

[9] R. H. Cannon, Jr., Dynamics of Physical Systems. New York: McGrawHill, 1967.

[10] T. Kailath, Linear Systems. Englewood Cliffs: Prentice-Hall, 1980.

[11] N. S. Nise, Control Systems Engineering. New York: Wiley, 1992.

[12] G. C. Goodwin, S. F. Graebe, and M. E. Salgado, Control System Design. Upper Saddle River: Prentice Hall, 2001.

[13] W. R. LePage, Complex Variables and the Laplace Transform for Engineers. New York: McGraw-Hill, 1961.

[14] H. H. Skilling, Electrical Engineering Circuits, 2nd ed. New York: Wiley, 1965.

[15] J. J. D'Azzo and C. H. Houpis, Linear Control System Analysis and Design: Conventional and Modern, 3rd ed. New York: McGraw-Hill, 1988.

[16] R. N. Clark, Control System Dynamics. Cambridge: Cambridge University Press, 1996.

[17] C. L. Phillips and R. D. Harbor, Feedback Control Systems, 4th ed. Upper Saddle River: Prentice Hall, 2000.

[18] M. F. Gardner and J. L. Barnes, Transients in Linear Systems. New York: Wiley, 1942.

[19] G. S. Brown and D. P. Campbell, Principles of Servomechanisms. New York: Wiley, 1948.

[20] H. Chestnut and R. W. Mayer, Servomechanisms and Regulating System Design. New York: Wiley, 1951.

[21] W. R. Ahrendt and J. F. Taplin, Automatic Feedback Control. New York: McGraw-Hill, 1951.

[22] G. J. Thaler and R. G. Brown, Servomechanism Analysis. New York: McGraw-Hill, 1953.

[23] J. G. Truxal, Automatic Feedback Control System Synthesis. New York: McGraw-Hill, 1955

[24] F. H. Raven, Automatic Control Engineering, 5th ed. New York: McGraw-Hill, 1995.

[25] C. M. Close and D. K. Frederick, Modeling and Analysis of Dynamic Systems, 2nd ed. New York: Wiley, 1995.

[26] D. S. Bernstein, Matrix Mathematics. Princeton: Princeton University Press, 2005.

[27] J. A. Aseltine, Transform Method in Linear Systems Analysis. New York: McGraw-Hill, 1958.

[28] O. J. M. Smith, Feedback Control Systems. McGraw-Hill, 1958.

[29] W. M. Siebert, Circuits, Signals, and Systems. Cambridge: MIT Press, 1986.

[30] H. R. Miller, D. L. Trumper, and K. H. Lundberg, "A brief treatment of generalized functions for use in teaching the Laplace transform," in Proceedings of the IEEE Conference on Decision and Control, San Diego, Dec. 2006.

[31] R. F. Hoskins, Delta Functions: An Introduction to Generalised Functions. West Sussex, England: Horwood Publishing Limited, 1999.

[32] R. K. Nagle and E. B. Saff, Fundamentals of Differential Equations, 3rd ed. Reading: Addison-Wesley, 1993.

[33] R. E. Williamson, Introduction to Differential Equations and Dynamical Systems. New York: McGraw-Hill, 1997.

[34] D. G. Zill and M. R. Cullen, Advanced Engineering Mathematics, 2nd ed. Boston: Jones and Bartlett, 2000.

[35] C. H. Edwards and D. E. Penney, Elementary Differential Equations with Boundary Value Problems, 5th ed. Upper Saddle River: Pearson Prentice Hall, 2004.

[36] W. E. Boyce and R. C. DiPrima, Elementary Differential Equations and Boundary Value Problems, 8th ed. Hoboken: Wiley, 2005.

[37] W. R. Evans, Control-System Dynamics. New York: McGraw-Hill, 1954.

[38] C. J. Savant, Jr., Basic Feedback Control System Design. McGraw-Hill, 1958.

[39] T. E. Fortmann and K. L. Hitz, An Introduction to Linear Control Systems. New York: Marcel Dekker, 1977.

[40] P. S. Buckley, Techniques of Process Control. New York: Wiley, 1979.

[41] Y. Takahashi, M. J. Rabins, and D. M. Auslander, Control and Dynamic Systems. Reading: Addison-Wesley, 1970. 

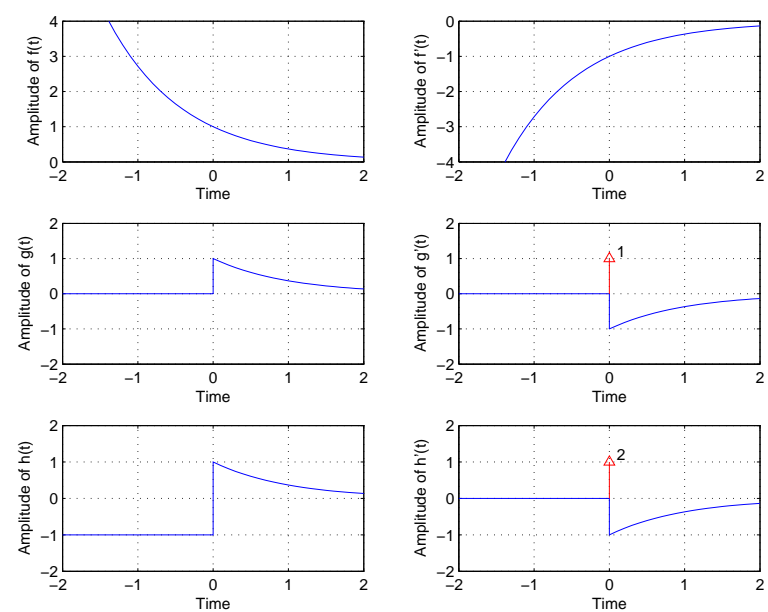

Fig. 5: Three functions $f(t)=e^{-a t}, g(t)=e^{-a t} u(t), h(t)=$ $e^{-a t} u(t)-u(-t)$, and their derivatives, plotted for $a=1$ and defined for all time. Impulses are represented by the red arrows, with the impulse area denoted by the number next to the arrowhead. Since all three functions are identical for positive time, they have identical unilateral Laplace transforms. However, their derivatives differ at the origin. Therefore the Laplace transforms of their derivatives also differ.

[42] B. C. Kuo, Automatic Control Systems. Englewood Cliffs: PrenticeHall, 1962.

[43] B. C. Kuo, Automatic Control Systems, 4th ed. Englewood Cliffs: Prentice-Hall, 1982.

[44] B. C. Kuo and F. Golnaraghi, Automatic Control Systems, 8th ed Hoboken: Wiley, 2003.

[45] K. Ogata, Modern Control Engineering, 4th ed. Englewood Cliffs: Prentice-Hall, 2002.

[46] K. Ogata, System Dynamics, 4th ed. Englewood Cliffs: Prentice-Hall, 2004.

[47] W. J. Palm III, Modeling, Analysis, and Control of Dynamic Systems, 2nd ed. New York: Wiley, 2000.

[48] G. H. Hostetter, C. J. Savant, Jr., and R. T. Stefani, Design of Feedback Control Systems. New York: Holt, Rinehart \& Winston, 1982.

[49] R. T. Stefani, B. Shahian, C. J. Savant, Jr., and G. H. Hostetter, Design of Feedback Control Systems, 4th ed. New York: Oxford University Press, 2002.

[50] R. C. Dorf, Modern Control Systems. Reading: Addison-Wesley, 1967.

[51] R. C. Dorf and R. H. Bishop, Modern Control Systems, 7th ed. Upper Saddle River: Prentice Hall, 1992.

[52] A. V. Oppenheim and A. S. Willsky, Signals and Systems. Englewood Cliffs: Prentice Hall, 1983

[53] A. V. Oppenheim and A. S. Willsky, Signals and Systems, 2nd ed. Upper Saddle River: Prentice Hall, 1997.

[54] G. F. Franklin, J. D. Powell, and A. Emami-Naeini, Feedback Control of Dynamic Systems. Reading: Addison-Wesley, 1986.

[55] G. F. Franklin, J. D. Powell, and A. Emami-Naeini, Feedback Control of Dynamic Systems, 3rd ed. Reading: Addison-Wesley, 1994.

[56] J. W. Grizzle, "Bookshelf: Linear Time-Invariant Systems by Martin Schetzen (book review)," IEEE Control Systems Magazine, vol. 24, no. 3, pp. 87-89, June 2004.

[57] P. M. Makila, "A note on the Laplace transform method for initial value problems," International Journal of Control, vol. 79, no. 1, pp. 36-41, Jan. 2006.

[58] H. M. James, N. B. Nichols, and R. S. Phillips, Theory of Servomechanisms. New York: McGraw-Hill, 1947.

\section{SIDE BAR \\ DisTINGUISHING $0^{-}, 0$, AND $0^{+}$}

While the unilateral Laplace transform is frequently associated with the solution of differential equations, the need to clearly distinguish $0^{-}, 0$, and $0^{+}$is independent of any dynamic-systems context. For a discontinuous function $f(t)$, the derivative $f^{\prime}(t)$ must be interpreted as the generalized derivative of $f(t)$, which includes the singularity function

$$
\left(f\left(t_{0}^{+}\right)-f\left(t_{0}^{-}\right)\right) \delta\left(t-t_{0}\right)
$$

at every point $t_{0}$ at which $f(t)$ is discontinuous. In particular, if $f\left(0^{-}\right) \neq f\left(0^{+}\right)$then the derivative includes a delta function at the origin.

In the following example, adapted from Problem 11.17 of [29], we apply the unilateral transform to three signals and their derivatives. This example clarifies that the need for using the Laplace transform (3) and properties (4) and (5) is really a matter of properly defining signals and their transforms, and is not fundamentally connected to the solution of differential equations.

Consider the three signals $f(t), g(t)$, and $h(t)$ defined for all time

$$
\begin{aligned}
f(t) & =e^{-a t}, \\
g(t) & =e^{-a t} u(t), \\
h(t) & =e^{-a t} u(t)-u(-t),
\end{aligned}
$$

which are plotted for the value $a=1$ in Figure 5. Since all three functions are nonsingular and agree for positive time, they have the same Laplace transform by means of (3). However, their derivatives include different impulse values at $t=0$, and thus the Laplace transforms of their derivatives must differ. Our choice of Laplace transform properties should give consistent and correct results when operating on these signals and their derivatives. The associated transforms are calculated below to show that this consistency is found. We also demonstrate the initial-value theorem in the context of these signals.

\section{A. Properties of $f(t)$}

Consider the function $f(t)=e^{-a t}$ with the pre-initial value $f\left(0^{-}\right)=1$. The Laplace transform of $f(t)$ is

$$
F(s)=\mathcal{L}_{-}\left\{e^{-a t}\right\}=\frac{1}{s+a} .
$$

The time derivative of $f(t)$ is

$$
f^{\prime}(t)=-a e^{-a t}
$$

and the Laplace transform of the time derivative is

$$
\mathcal{L}_{-}\left\{-a e^{-a t}\right\}=\frac{-a}{s+a} .
$$

Using the derivative rule (4)

$$
\mathcal{L}_{-}\left\{f^{\prime}(t)\right\}=s F(s)-f\left(0^{-}\right)=\frac{s}{s+a}-1=\frac{-a}{s+a}
$$

produces the same result. The results from the initial-value theorem are

$$
f\left(0^{+}\right)=\lim _{s \rightarrow \infty \cdot 1} \frac{s}{s+a}=1
$$

and

$$
f^{\prime}\left(0^{+}\right)=\lim _{s \rightarrow \infty \cdot 1} \frac{-s a}{s+a}=-a .
$$

The time-domain and Laplace-domain calculations are thus consistent. 


\section{B. Properties of $g(t)$}

The function $g(t)=e^{-a t} u(t)$ has an associated pre-initial value $g\left(0^{-}\right)=0$. The Laplace transform of $g(t)$ is the same as for $f(t)$, namely,

$$
G(s)=\mathcal{L}_{-}\left\{e^{-a t} u(t)\right\}=\frac{1}{s+a} .
$$

However, the time derivative now includes an impulse at the origin

$$
g^{\prime}(t)=\delta(t)-a e^{-a t} u(t) .
$$

The Laplace transform of this time derivative is

$$
\mathcal{L}_{-}\left\{g^{\prime}(t)\right\}=1-\frac{a}{s+a}=\frac{s}{s+a},
$$

which is different from the result (18) above. Using the derivative rule (4),

$$
\mathcal{L}_{-}\left\{g^{\prime}(t)\right\}=s G(s)-g\left(0^{-}\right)=\frac{s}{s+a}-0=\frac{s}{s+a},
$$

we obtain the correct result. The initial-value theorem gives

$$
g\left(0^{+}\right)=\lim _{s \rightarrow \infty \cdot 1} \frac{s}{s+a}=1,
$$

producing the value at $t=0^{+}$. We can also apply the more general initial-value theorem to the transform of the derivative as follows. Expanding out the nonsingular part of the transform gives

$$
G(s)=1-\frac{a}{s+a} \equiv 1+\tilde{G}(s),
$$

and thus

$$
g^{\prime}\left(0^{+}\right)=\lim _{s \rightarrow \infty \cdot 1} s \tilde{G}(s)=-a,
$$

which is the correct value.

\section{Properties of $h(t)$}

Finally consider the function

$$
h(t)=e^{-a t} u(t)-u(-t)= \begin{cases}-1, & t<0, \\ e^{-a t}, & t>0,\end{cases}
$$

which has an associated pre-initial value $h\left(0^{-}\right)=-1$. The Laplace transform of this signal is the same as the Laplace transforms of $f$ and $g$. Computing the time derivative gives

$$
h^{\prime}(t)=2 \delta(t)-a e^{-a t} u(t) .
$$

The Laplace transform of this time derivative is

$$
\mathcal{L}_{-}\left\{h^{\prime}(t)\right\}=2-\frac{a}{s+a}=\frac{2 s+a}{s+a} .
$$

which is different from the results (18) and (19) above. Using the derivative rule (4),

$$
\mathcal{L}_{-}\left\{h^{\prime}(t)\right\}=s H(s)-h\left(0^{-}\right)=\frac{s}{s+a}+1=\frac{2 s+a}{s+a},
$$

gives the correct result. Finally, the initial-value theorem gives a correct result for both $h$ and its derivative, $h\left(0^{+}\right)=1$ and $h^{\prime}\left(0^{+}\right)=-a$, although we don't show the details here.

The formulas (3), (4), and (5) give consistent results. We hope that the signal examples presented above help to clarify the need for and application of these formulas.

\section{THE AUTHORS}

Kent H. Lundberg (klund@mit.edu) received his Ph.D. in electrical engineering from the Massachusetts Institute of Technology in 2002, where he was a lecturer with the Department of Electrical Engineering and Computer Science from 2002 to 2005. His research interests include the application of control theory to problems in analog circuit design and the development of educational toys (such as lecture demos, web applets, and low-cost lab kits) for students in control engineering. He is the associate editor for history of IEEE Control Systems Magazine. He consults for several industry corporations and organizations, and he collects old textbooks on radar, nuclear energy, analog computing, and control. He can be reached at MIT Lincoln Laboratory D-362A, 244 Wood Street, Lexington, MA 02420 USA.

Haynes R. Miller is professor of mathematics at the Massachusetts Institute of Technology. His research specialty is algebraic topology. Over the past decade he has taught the major ordinary differential equations course several times, and has developed a series of "Mathlets" (http://www-math.mit.edu/daimp) for use in lecture demonstrations and in homework assignments. He is a Margaret MacVicar Faculty Fellow and the 2006 School of Science winner of the Graduate Student Council teaching award.

David L. Trumper joined the MIT Department of Mechanical Engineering in August 1993 and holds the rank of professor. He received the B.S., M.S., and Ph.D. degrees from MIT in electrical engineering and computer science, in 1980, 1984, and 1990, respectively. Professor Trumper's research centers on the design of precision electromechanical systems with a specialization in mechatronics, precision motion control, highperformance manufacturing equipment, and magnetic suspensions and bearings. He is a member of the IEEE, ASME, and ASPE (past-president), is an associate editor of Precision Engineering, and is a corresponding member of CIRP (The International Academy for Production Engineering). 